\title{
OCCUPATIONAL TRANSITIONS: A COHORT ANALYSIS OF TRANSITIONS BETWEEN OCCUPATION CLASSES 1981 TO 2006
}

\author{
Robert Didham and Kirsten Nissen
}

Statistics New Zealand

\section{Introduction}

This paper is limited to presenting an initial look at the New Zealand Linked Census. The intention here is limited to describing the NZ Linked Census, providing an example of how this new dataset can be used in the wider context of labour market themes, and promoting the dataset as a powerful tool for new and ground breaking longitudinal research.

\section{New Zealand Linked Census (NZLC)}

The New Zealand Linked Census links censuses for the period 1981 to 2006.

A full description of the datasets and details on how to access the data may be found here:

- http://www.stats.govt.nz/methods/data-integration/data-integrationprojects/linking-censuses-nzlc-1981-2006.aspx

Below is a brief summary to contextualise the discussion which follows.

Each census is linked to its neighbouring census to create five pairs of linked censuses (1981-1986, 1986-1991, 1991-1996, 1996-2001, 2001-2006). Because each pair has a common boundary with at least one other pair, these are linkable to each other. It is possible to construct longitudinal series of varying lengths for people who have been linked across multiple censuses.

Each pair contains over 2 million linked records, with a total of around 4.6 million records linked at least once in the five pairs. For over 0.6 million records the links cover the whole period of 19812006. For a link to be made for the whole period, a person has to have been born prior to census in March 1981, have been in New Zealand and enumerated at every census from 1981 up to and including 2006. One constraint is that someone may be linked in, say, two pairs covering 19811991 and again in the 2001-2006 pair, as may happen for many people who were either out of New Zealand or not enumerated for any of the intervening censuses. The possibility of linking between these two sets of links has not yet been investigated using migration data and family relationship information - in other words people may enter and drop-out, but if they re-enter in the current datasets this re-entry is currently not identified. 
Figure 1 provides graphically a broad overview of drop-out. The most striking feature is the consistency in the size of the groups by number of links made. For example, the number of records linked across two census points is consistently around 2 million regardless of which census is being considered. Similarly there are around 1.5 million linked across three census points, and so on through to those linked across 6 census points (where the latest census can only be 2006). Note that these are not cumulative: a record linked across 3 census points (e.g. if the latest census is 2001) will be present in two sets of those linked across 2 census points (in this case 1991-1996 and 1996-2001).

Figure 1: Number of linked census points by the latest linked census, NZLC.

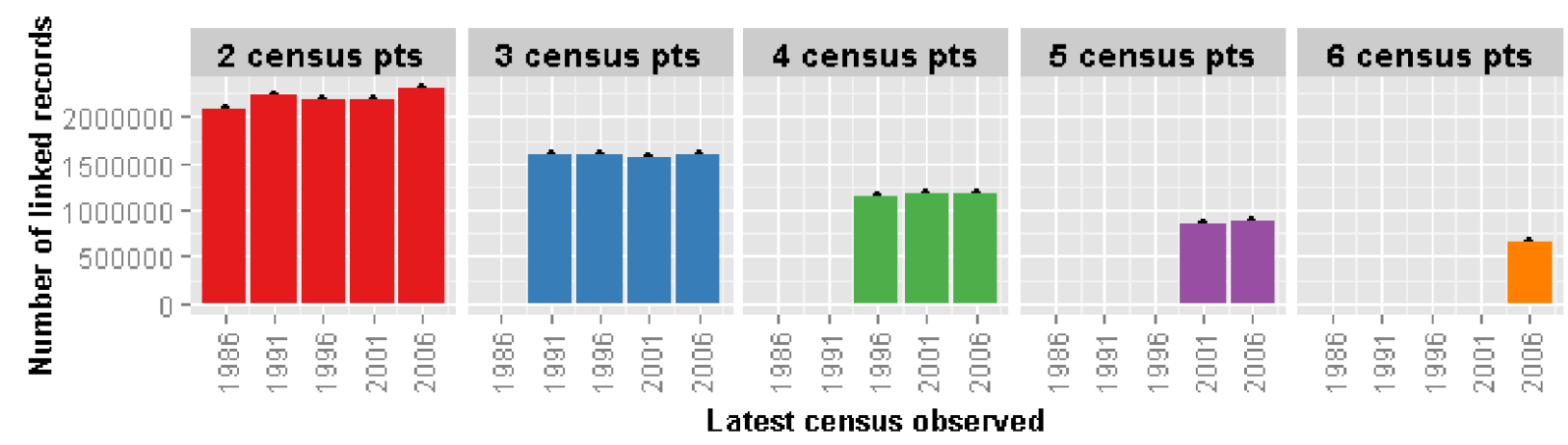

\section{Link rate by age and sex}

A major element in the drop-out pattern relates to differing link rates by both age and sex. Young adults have least chance of being linked (figure 2), with link rates falling to almost 50 percent for males, though females are substantially more successfully linked. This creates biases and limits our knowledge of the precursor characteristics when we work with cohorts - we know about a smaller proportion of the population and those who are linked may have quite different characteristics from the not-linked population (for example people who move frequently are more likely not to be linked and people who are overseas for one or more of the censuses cannot be linked back for the whole period). Conversely if our interest is in the older age groups, the high link rate for the midadult and the older age groups means we have very good data to work with.

Hence if we are considering occupational transitions for the people in mid-career in 1981 to explore the career, retirement or health outcomes later in their lives, the data is of high enough quality and coverage to enable very detailed analysis. However if we are interested in the progress of people through education and work force entry to examine outcomes of policy interventions on mid-career occupations we need to be aware that we are missing links for a significant portion of our subject population and we need to consider biases introduced in the linking process and the effect of missing data. 


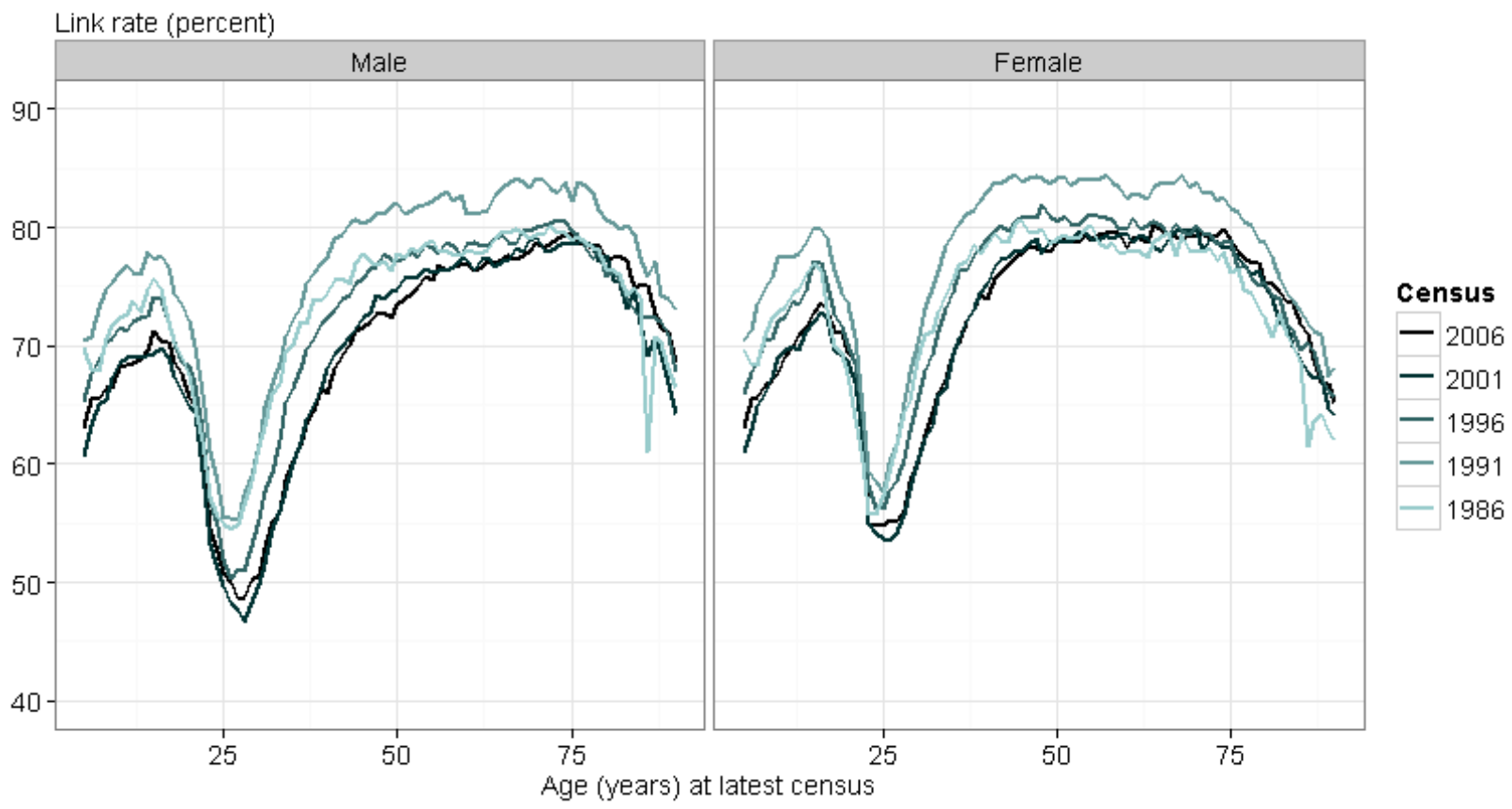

Occupation transitions - definition and classification

Turning now to an example of how the longitudinal census might be used, we look at occupation transitions. The data used here is restricted to the subset of the records that have been linked for the whole period 1981-2006 and were employed at both 1981 and 2006 censuses. Occupation classifications have changed dramatically over this period but it has been possible to recode occupation information successfully to level 1 of NZSC099. The categories at this level are:

1 Legislators, administrators, and managers

2 Professionals

3 Technicians and associated professionals

4 Clerks

5 Service and sales workers

6 Agriculture and fishery workers

7 Trades workers

8 Plant and machine operators and assemblers

9 Elementary occupations

Ideally for this study all transitions between each census point would be considered to see whether different pathways had different outcomes. The main problem with this approach is that for all who were linked and had an occupation listed at least twice (otherwise there would be no transition involved) there are still over 116,000 different recorded pathways, making it difficult to identify patterns and trends, and almost impossible to display graphically. Interestingly, for males there are 67,900 pathways and for females 67,800 , implying a significant proportion of pathways are unique to either males or females, potentially showing significant gendering in the work force.

For the present purpose an occupation transition is defined as a change of occupation in a level 1 category listed above to an occupation in a different level 1 category listed above. We have not yet considered progressions and transitions within categories. Moreover, when we talk about movement of people "up" and "down" hierarchies we are talking in terms of the order of the 
categories as set out in the NZSCO classification. We are not implying in any way that any category (e.g. managers) have a greater value than any other (e,g, professionals).

Bias in linking can also be seen across occupation groups. Figure 3, using the 2006-2001 pair as an example, shows the link rates for the employed population. Two features are noteworthy: there are generally higher link rates for females and link rates tend to decline down the occupation classification hierarchy independently of both age and sex. In part the differences in link rate is influenced by a combination of factors such as geographic location (especially by NZ Deprivation Index Area), geographic mobility and qualifications. This has implications for any analysis of occupational mobility because the missing information is not uniformly missing across the parameters we are most interested in. Thus, the data presented here is raw - that is, it has not been adjusted for bias due to differential linking across the hierarchy - and therefore needs to be treated with due caution when drawing inferences. Moreover, figure 3 only shows the 2001-2006 pair and it should not be, ipso facto, assumed that the pattern above applies equally to other pairs. However, similar variations by age, sex and occupation can be demonstrated for other pairs which suggest that the other pairs have the same characteristics.

Figure 3: Link rates, level 1 occupation groups, 2001-2006 linked census pair, by age and sex, NZLC

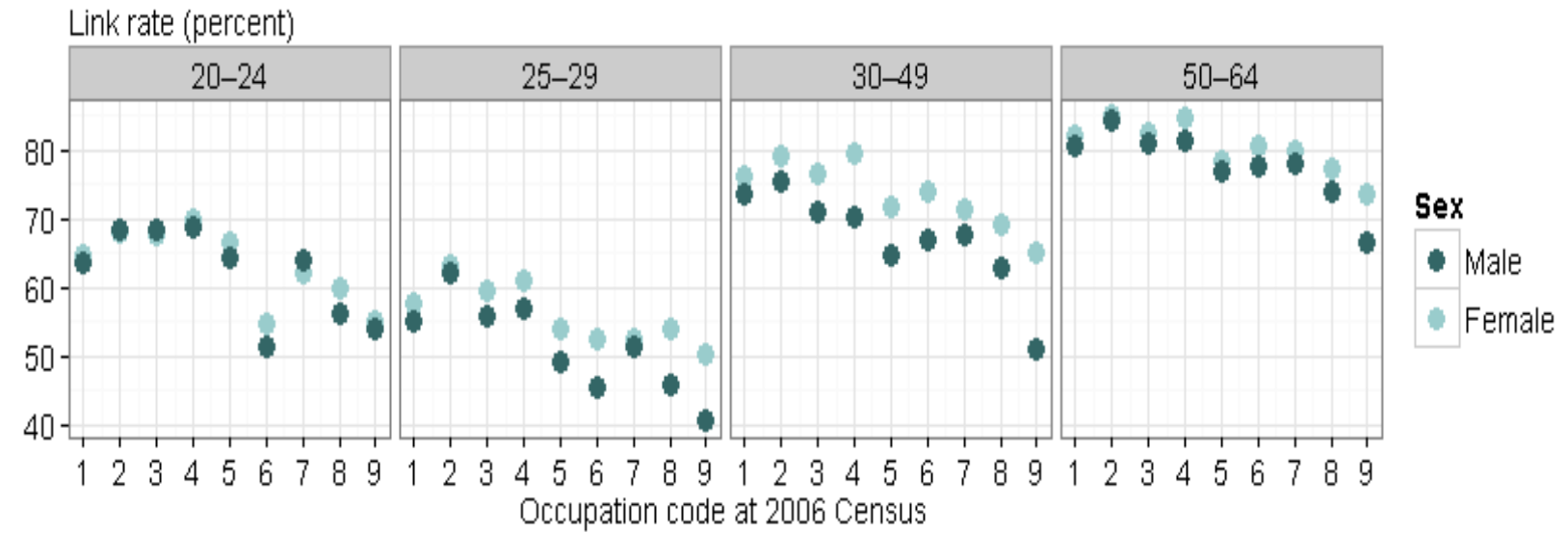

\section{Two questions and research potential}

This paper takes a slightly blunt look by simply comparing the 1981 occupations of people linked for the whole period with their 2006 occupations, and asks two questions with respect to occupation transitions:

1. Where did the people in occupation groups in 2006 come from in 1981 ?

2. Where did people in occupation groups in 1981 end up in 2006 ?

While occupational transitions would ideally require investigation of sub-national differences and examine changes between each pair to identify the relationship and timing. This would also involve considering the relationship between occupational mobility and socio-political and economic environments, and this data source certainly provides the framework for such a detailed study, but that remains to be done. We are only looking here at level 1 of the occupation classification and we therefore need to remember that within each of the level1 groupings there is huge diversity. 
Two age groups are selected: people aged 20-24 years in 1981 and those aged 35-39 years in 1981 . People aged 20-24 in 1981 are in their early career in 1981 and are in their mid-career 2006 (45-49 in 2006 - and born 1956-1961). Conversely, people in mid-career 1981 (aged 35-39 years) are in their later career path (60-64 in 2006 and born 1941-46) in 2006. For the current purpose we have just used those linked for the whole period, but we could also possible have looked at birth cohorts (e.g. in the case of the 20-24 year olds, all those born 1956-1961) and include those only linked for only some part of the period.

The analysis is further restricted here to looking at two specific groups: (1) those who were "legislators, administrators and managers" in 2006 and (2) people who were "technicians and associated professionals" in 1981.

As noted above, this is just a subset of people and by definition they are people who did not have time out of NZ, or missed a census for any other reason, or who could not be linked at any point. Drop-out of this type introduces bias because we know that people who are missed or have periods overseas differ from those who do not. We also need to consider bias caused by non-linked people, because these also tend to be more mobile and harder to link. Bias analysis is currently being considered by a team at Auckland University.

\section{Legislators, administrators and managers in 2006 - what were they doing in 1981?}

One of the questions we might wish to ask is whether the people who are in managerial types of occupations in 2006 had started out as managers or were drawn from different occupation groups.

Males and females have different pathways. Part of the difference is because of the highly gendered nature of employment in 1981, with the main pools of female skill in different occupation areas than males. The managerial occupation group also cover a wide spectrum of industry groups, each with different gender profiles.

Figure 4: Occupation groups in 1981 of males who were legislators, administrators and managers in 2006, aged 20-24 years and 35-39 years in 1981, NZLC.

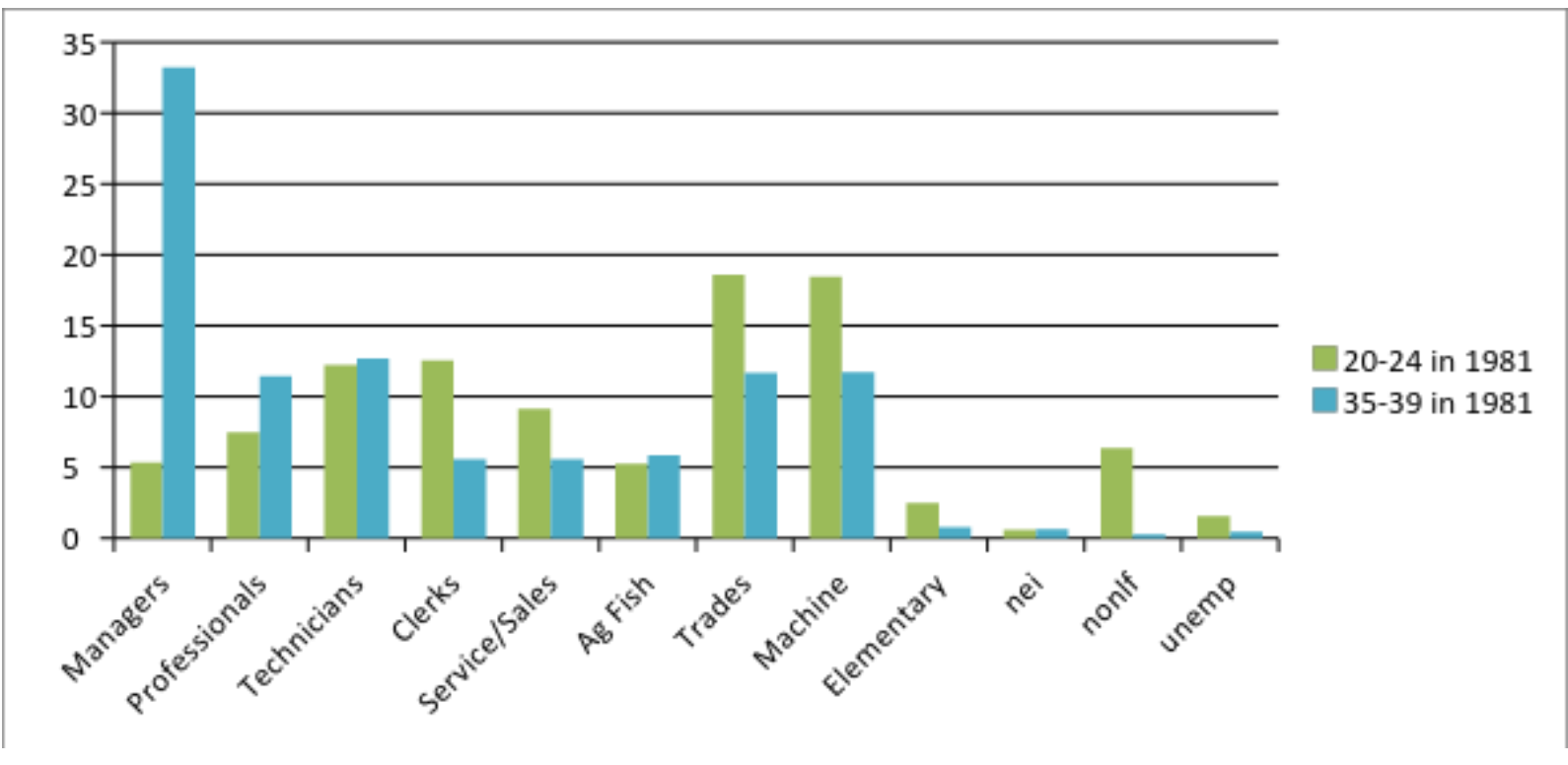


Males who were in managerial roles in 2006 were spread widely across all occupation groups in 1981, but tend to have been in occupation areas requiring education or advanced skill bases (machine operators, trades, technicians) or to have been in administrative roles (clerical). There is a difference seen, in figure 4, between the two age groups. Among those aged 35-39 years in 1981, a third were already mid-career managers in 1981, with the majority of the others moving in from technical and trades occupations. The early-career group (20-24 years) show that these were also the pathways for people who had moved into mid-career management by 2006, suggesting a continuing diversity in occupation progression. Many of the males who had moved from service and sales were ex-military.

Figure 5: Occupation groups in 1981 of females who were legislators, administrators and managers in 2006, aged 20-24 years and 35-39 years in 1981, NZLC.

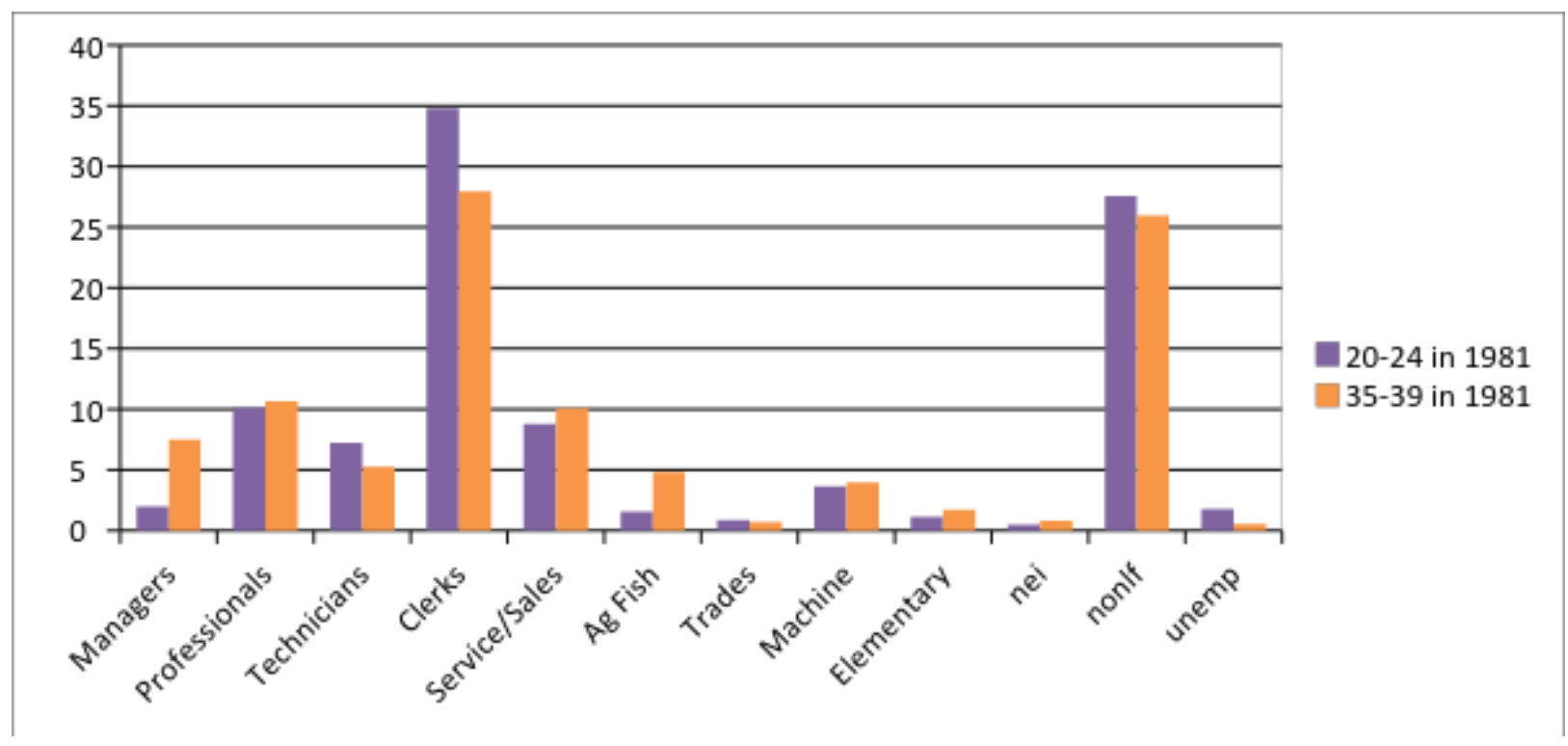

Females show a markedly different pattern from males (figure 5). Very few women who were managers in 2006 had been managers in 1981. The vast majority of those working in 1981 were clerical workers in 1981. Moreover, a quarter of each age group were not in the labour force. Most of the women in this category were rearing children, either as young mothers or with young children at home, and most were partnered. There are two distinct groups of women here: those who had children before they were 25 years of age and those who were mothers to young children in the 35-39 group.

The difference between males and females provides evidence of the very different pathways that males and females took to where the labour market is now - notably with the feminisation of the political and administrative heartland. But it also shows the interlinking of work and society, with the relationship between childbearing and the labour market. Interestingly the pattern is very similar for both early and mid-career women suggesting that a question requiring further research is how the socio-political environment affected the timing of transitions into management for women - for example, if the pathways are similar for both age groups, as we see here, did the feminisation of management occur in concert with EEO legislative initiatives at a similar time and at a similar pace for different age groups, or birth cohorts, or were all ages groups affected at similar 
times. A closer look at transitions at various points in time across the linked pairs of censuses would be needed to answer this question.

\section{Technicians and associated professionals in 1981 - where did they end up?}

With managers, the question we set ourselves was how they got there. We can turn this question upside down by asking where people moved to. One group of interest because of the ongoing concern for skill shortages and loss of expertise in technical areas, is the technicians and associated professionals group. Well over 10 percent of the males and around 5 percent of the females who were in the legislators, administrators and managers group in 2006 had come from this group, but this does not tell us what proportion of the technicians and associated professionals were lost to management, nor what happened to the rest. We therefore pose the question: where were those employed as technicians and associated professionals in 1981 found in $2006 ?$

This group of occupations generally include highly skilled people, but also it is also a very diverse group of occupations with high levels of internal gendering. Engineering tended to be male dominated in 1981, with health and life sciences very highly female dominated. This group covers such diverse areas as finance, human resources and writing.

Males (figure 6) and females (figure 7) show quite different patterns. The younger women (aged 20-24 years in 1981) were in their late 40s in 2006, many with families at home, were either out of the work force (19 percent) or were still in the same occupation group (23 percent) or had moved up into the professional occupation group (19 percent).

Nearly a third of the older group had also dropped out of the work force. Some may be grandmothers caring for children, or in the voluntary work sector. Surprisingly, there is a high proportion of men ( 21 percent) in the same situation. We have not considered here whether there is a relationship between which occupations or industries contributed to this drop-out, and it may be related to loss of opportunities in the employment sector, especially in the physical sciences and social sciences.

Figure 6: Occupation groups in 2006 of males who were technicians or associated professionals in 1981, aged 20-24 years and 35-39 years in 1981, NZLC. 


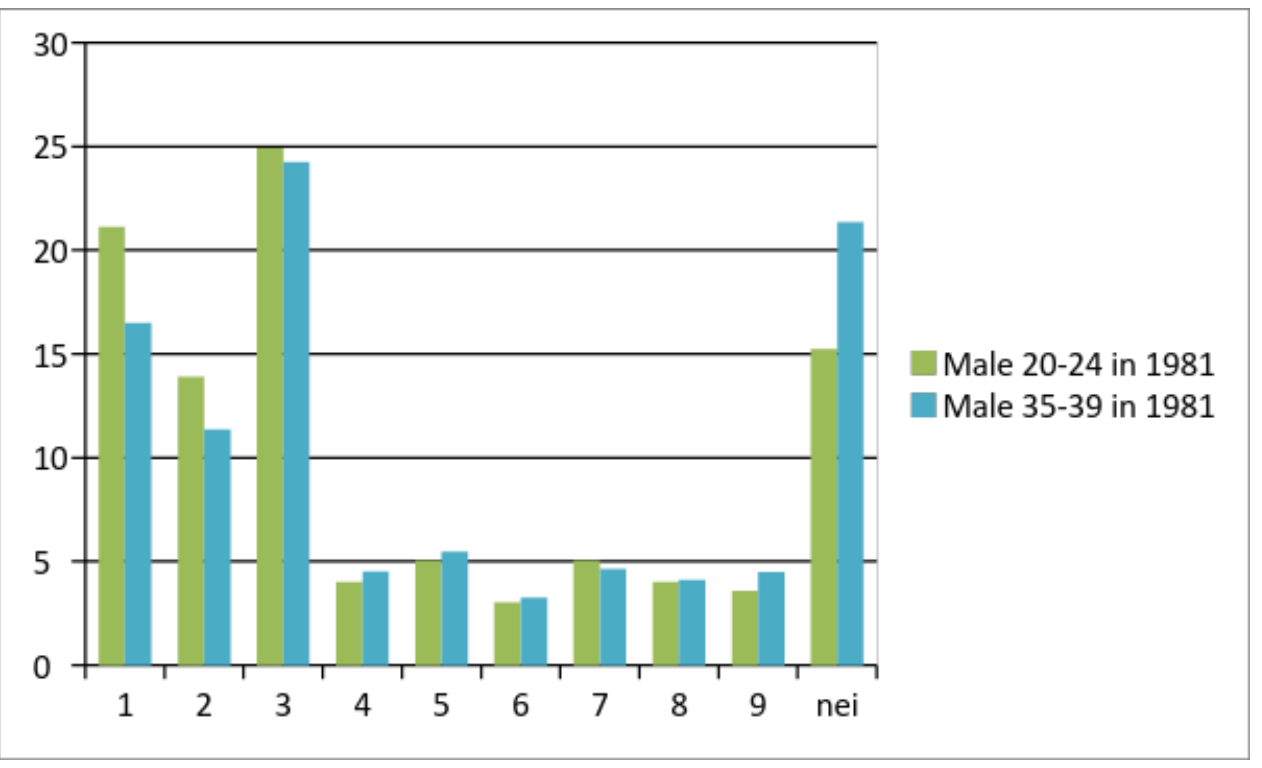

Figure 7: Occupation groups in 2006 of females who were technicians or associated professionals in 1981, aged 20-24 years and 35-39 years in 1981, NZLC.

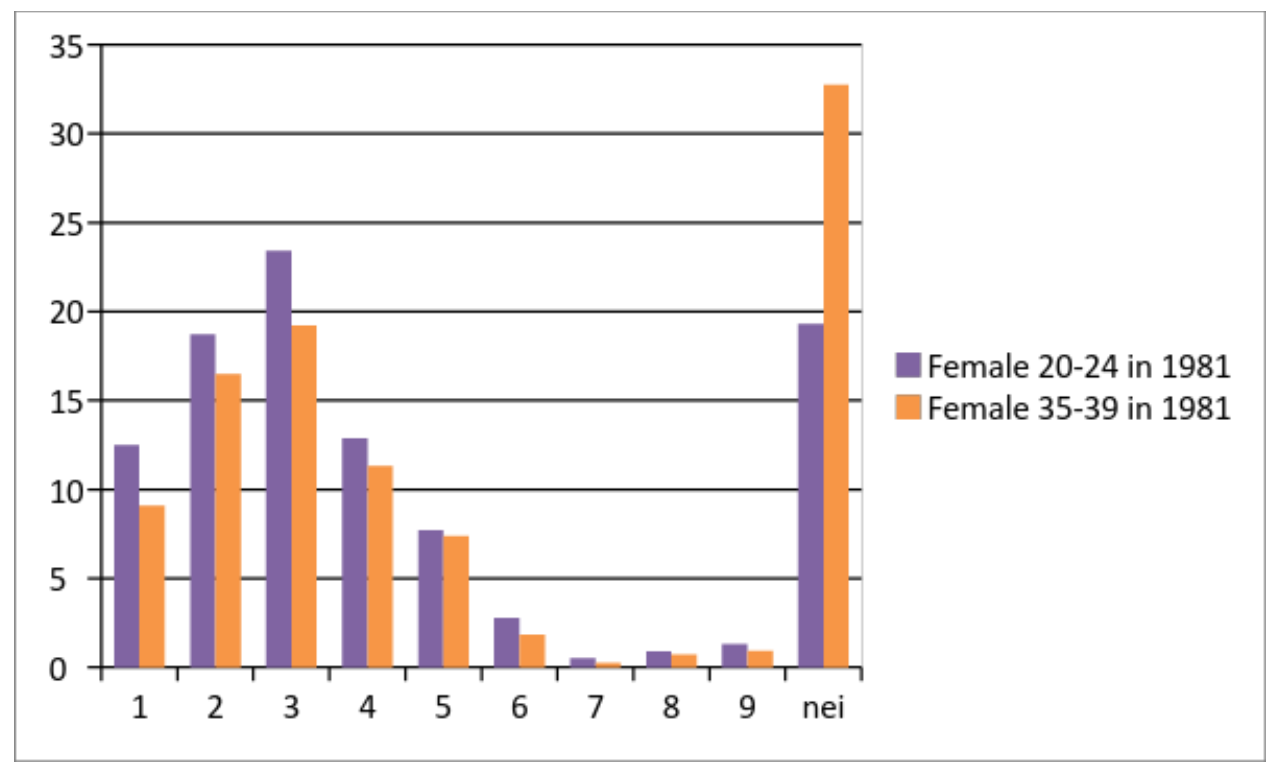

Pattern is different for those still working. Men are more likely than women to be either in the same category or moved up to professionals or out to management. Women are more likely to have moved into the professional group or, to a lesser extent, across into the clerical and care/service groups, often changing their roles within their areas of technical expertise. Women are much more likely than men to have moved into caregiving and community services.

\section{Ethnicity and occupational transitions}

Ethnicity and occupation clustering is a topic that frequently gains attention. One of the difficult questions, though is how this clustering happens and why it happens. However, most of the work 
done with census data that is not linked has worked with quasi-cohorts, on the assumption that the groups contain sufficiently high proportion of the same people to be comparable. With linked census we are dealing with real cohorts, and this has shown that quasi-cohort approach is at best an approximation because of the ways in which people change over time. For example, people change their ethnic identification in census frequently, either by changing, or by adding or dropping, the ethnicities they identify with at the time of the census. This is further complicated by changes within and between other collections that we might want to use in research (such as health or education data).

In this section, we look at people of Māori as an example of ethnicity and because the field of Māori development is of vital policy interest. Ethnic mobility, the phenomenon of people changing ethnic identification over time, is therefore an important component of any longitudinal analysis based on ethnicity. It is also a topic of current research being carried out by one of the current authors (Didham). Since most of the statistics in New Zealand are produced both by total population as well as by/for/about Māori, the way in which the data represents Māori and how this representation changes over time matters a very great deal to how we understand what we are seeing in the data.

In the linked census, several things affect the data we have. Linking young adults (especially males) is difficult, Māori young adults have lower link rates, and across six censuses the propensity to be missed at least once is high. For this reason a fuller picture looking at each census pair will give a better understanding of the processes and how this relates to the social and economic historical environments. However, here we follow the same pattern as we did for the total population, using 1981 and 2006 as end points. Hence, ethnic mobility here is simply defined on the basis of these two end points - many more people in the data sets may have been Māori at any of the intermediate points but not at either end point, or at all points except for one of the end points. It must be stressed that this is only an initial and partial view of current work only in its early stages of development.

For Māori ethnic mobility defined as the difference between 1981 and 2006 for the population linked at all points, the major transition across the boundary between people with other ethnicities as well as Māori ethnicity and those without Māori ethnicity. This was surprising from what we know about other data is how few people transition between what used to be called "sole" Mãori and "mixed" Māori (these terms are used here cautiously because of the colonial overtones). It appears that people tend to transition into and out of identifying as Māori in the census and it is the "other" ethnicities that are sticky (be they Pacific or European ethnicities, or both). This will be reported on at a later date, after further research has been completed.

\section{Maori male managers in 2006 - pathways to management}

The first difficulty to solve when we are dealing with data which changes is what the correct denominator is. For outflows, clearly the best is the population at the start of the process to measure the proportion flowing out. Similar for the population flowing in the end point, the end population is a suitable denominator. However if the interest is in both inflows and outflows in relation to some other feature, neither is satisfactory. One option is to construct the denominator 
to include both - in this case people who were Māori in 1981 and/or people who were Māori in 2006. This is often referred to as "ever Māori".

For several reasons, "ever Māori" is conceptually rather problematic but is used in some quarters, and here does form a useful denominator. Again, it is stressed that we are looking purely at Māori in 1981 versus Māori in 2006 and this does not imply anything about intervening censuses for this paper. There are many theoretical matters associated with this, not least of which is the question of the extent to which Māori "ethnic origin" as measured in 1981 is similar to Māori ethnicity as measured in2006 (and here it is total Māori in both cases and as consistent as we can make it for both points in time).

Figure 8 demonstrates both the complexity of the data and the diversity of change for Māori both in occupation and in ethnic identification. This is shows a comparable set of information to figure 4, but is about people who had reported their ethnicity, or one of their ethnicities, as Māori in either the 1981 census or the 2006 census or in both the 1981 and 2006 censuses. Only males are presented here as an example.

We need to be very cautious about small numbers and even more cautious about inherent biases in the data, as discussed above. But we can very tentatively note some of the features which will be included in future research. These have been deliberately left in note form to indicate that they are still "live" thoughts which need fleshing out in a future paper

1. The key difference is between those who were managers in BOTH 2006 and 1981 and those who were managers in 2006 and machine operators in 1981. The former group tended to "lose" Māori, the latter "gain" Māori. Why this is important is that it might be the smoking gun of institutional racism (it might not be too - needs more work - and we only know WHAT people told us or more importantly did not tell us, we do not know WHY)

2. Implicit in these graphs are a variety of social, economic and political contexts and the relationship between Māori development programmes and policies and the labour market response.

3. As Tahu Kukutai noted in a recent conference: "there is a growing recognition that such numbers are not merely the products of value-neutral data collection activities, but also reflect political processes and group struggles for recognition"

4. Comparing these patterns with the earlier slide for the population as a whole it is clear that the pathways into management for Māori are much more diverse than for people in other groupings of ethnicities - and the pathways are also quite different. It remains to see whether the pathways for females differ. Both are topics for much more work.

Figure 8: Occupation groups in 1981 of males of Māori ethnicity who were legislators, administrators and managers in 2006, aged 20-24 years and 35-39 years in 1981, NZLC. 
Māori in 1981 but not in 2006

Māori in 2006 but not in 1981
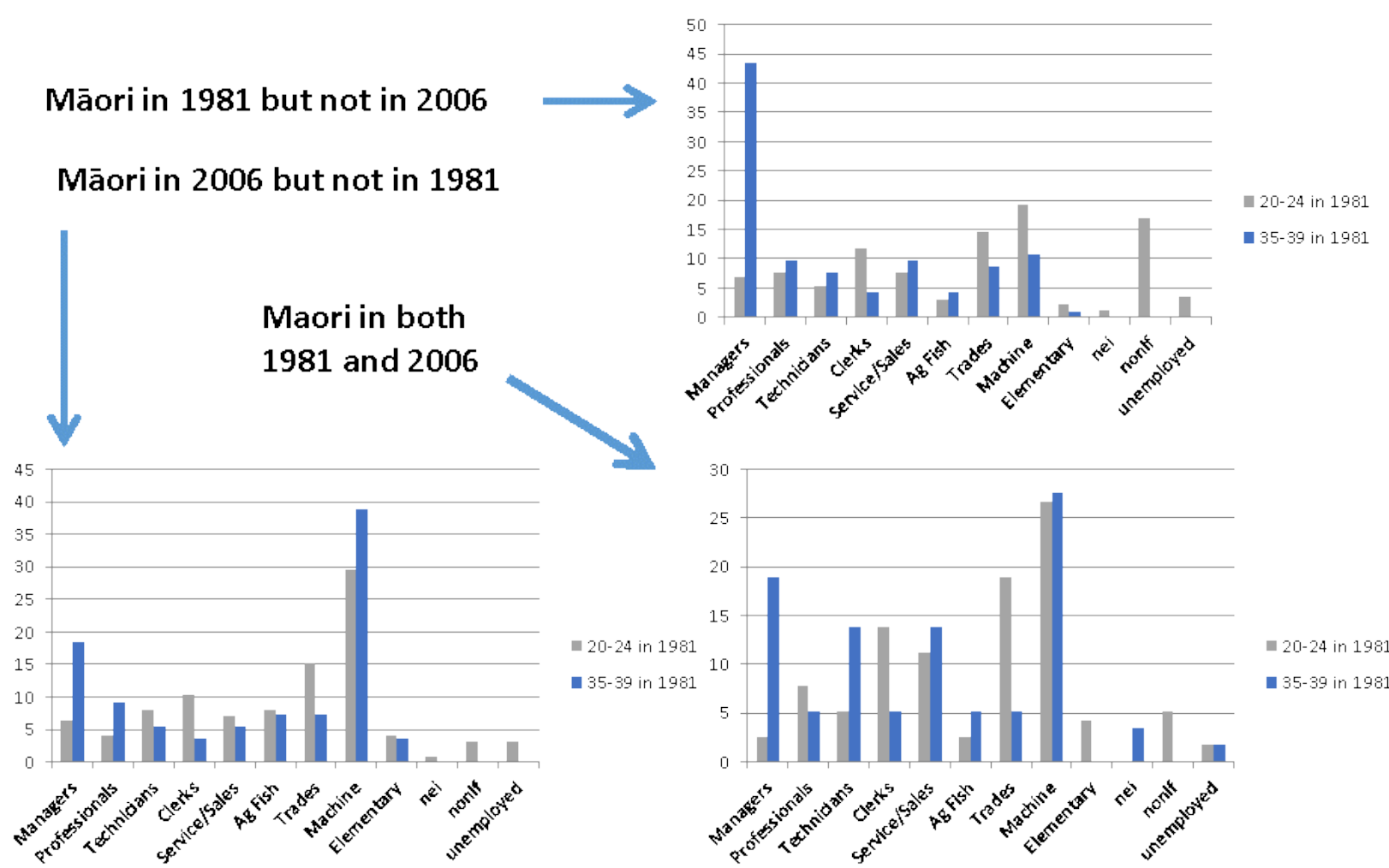

\section{Future potential and research suggestions}

This is a grammarian's heading if ever there was one.

This paper has just touched on the edges of a potentially very large fruitful field for further research. At the conference this was touted as a kind of "extended ad-break" for the New Zealand Linked Census, and this is a fair description because it both promoted this important new data source and set out to illustrate a practical way in which it can be used to advance $\mathrm{k}$ knowledge in the labour market area.

It of course has many more applications across the economic and social arenas. Among the potential topics of interest in the labour market research field which have been touched upon in this paper are:

- Gendered labour market transitions

- Relationship between labour market performance and socio-historical change

- Credentialism and "qualification inflation"

- Effect of migration flows on pathways

- Effect of childbearing and childrearing

- Linked censuses enable real cohort analysis

This paper has restricted itself to two very simple and high level questions to illustrate the power of the linked census. It also just looked at two age groups, hinting at the themes listed above, but there is a lot more of interest going on in other age groups as well, especially in relationship to family structural changes, education, re-training, second and third careers, modifications to gender 
balances and the role of migration. There is a very complex web of exchanges which we expect to emerge once we start to unpick the data by ethnicity and birthplace.

We have not looked at all closely at transitions, both sub-nationally and between each pair, to identify the nature and timing between occupational mobility and socio-political and economic environments. This would seem to be an area suitable for thesis topics. Potentially, this research could sit alongside other data sources such as HLFS and other household surveys to look at intercensal changes.

Sub-national research could extend the themes listed above to consider the role of the overseas born, for example does migration affect direction, location and timing of changes or do these changes have an impact on migration. Similarly, we could investigate whether the pathways are different for Maori and for women, and if so why this should be so. For example the differences may be related to geographies or to social factors, with implications for how we distinguish aspects that relate to ethnicity from aspects that relate to other factors.

By far the greatest value in the dataset is the capacity to analyse real cohorts in a number of ways. For example, if we consider how the patterns differ for different cohorts (e.g. born pre1920, 1921 to 1935,1936 to 1950,1951 to 1965,1966 to 1980 , and 1981 and later) we would be better able to distinguish cohort from age patterns. This would in turn enable a close examination of the role of economic, education and migration policies in labour market outcomes. 